\title{
Sample return of interstellar matter (SARIM)
}

\author{
Ralf Srama • Thomas Stephan • Eberhard Grün • \\ Norbert Pailer • Anton Kearsley • Amara Graps • \\ Rene Laufer • Pascale Ehrenfreund • \\ Nicolas Altobelli • Kathrin Altwegg • \\ Siegfried Auer • Jack Baggaley • Mark J. Burchell • \\ James Carpenter • Luigi Colangeli • \\ Francesca Esposito • Simon F. Green • \\ Hartmut Henkel • Mihaly Horanyi • \\ Annette Jäckel • Sascha Kempf • Neil McBride • \\ Georg Moragas-Klostermeyer • Harald Krüger • \\ Pasquale Palumbo • Andre Srowig • Mario Trieloff • \\ Peter Tsou • Zoltan Sternovsky • Oliver Zeile • \\ Hans-Peter Röser
}

Received: 24 November 2007 / Accepted: 10 March 2008 / Published online: 3 May 2008

(C) The Author(s) 2008

\begin{abstract}
The scientific community has expressed strong interest to re-fly Stardustlike missions with improved instrumentation. We propose a new mission concept, SARIM, that collects interstellar and interplanetary dust particles and returns them to Earth. SARIM is optimised for the collection and discrimination of interstellar dust
\end{abstract}

R. Srama $(\bowtie) \cdot$ E. Grün $\cdot$ S. Kempf $\cdot$ G. Moragas-Klostermeyer Max-Planck-Institute Nuclear Physics, Heidelberg, Germany

e-mail: ralf.srama@mpi-hd.mpg.de

R. Srama • R. Laufer • O. Zeile $\cdot$ H.-P. Röser

IRS, University Stuttgart, Stuttgart, Germany

T. Stephan

University of Chicago, Chicago, USA

E. Grün • M. Horanyi · Z. Sternovsky

LASP, University Colorado, Boulder, USA

N. Pailer

Astrium GmbH, Friedrichshafen, Germany

A. Kearsley

Natural History Museum, London, U.K.

A. Graps

INAF-IFSI, Roma, Italy 
grains. Improved active dust collectors on-board allow us to perform in-situ determination of individual dust impacts and their impact location. This will provide important constraints for subsequent laboratory analysis.

The SARIM spacecraft will be placed at the L2 libration point of the Sun-Earth system, outside the Earth's debris belts and inside the solar-wind charging environment. SARIM is three-axes stabilised and collects interstellar grains between July and October when the relative encounter speeds with interstellar dust grains are lowest (4 to $20 \mathrm{~km} / \mathrm{s}$ ). During a 3-year dust collection period several hundred interstellar and several thousand interplanetary grains will be collected by a total sensitive area of $1 \mathrm{~m}^{2}$. At the end of the collection phase seven collector modules are

P. Ehrenfreund

University Leiden, Leiden, The Netherlands

N. Altobelli

JPL, Pasadena, USA

K. Altwegg • A. Jäckel

University Bern, Bern, Switzerland

S. Auer

A\&M Associates, Basye, V.A., USA

J. Baggaley

University Canterbury, Christchurch, New Zealand

M. J. Burchell

University of Kent, Canterbury, Kent, UK

J. Carpenter

University Leicester, Leicester, U.K.

L. Colangeli $\cdot$ F. Esposito

INAF-Osservatorio Astronomico di Capodimonte, Napoli, Italy

S. F. Green • N. McBride

Open University, Milton Keynes, U.K.

H. Henkel

von Hoerner \& Sulger GmbH, Schwetzingen, Germany

S. Kempf

University Braunschweig, Braunschweig, Germany

H. Krüger · P. Tsou

MPS, Katlenburg-Lindau, Germany

A. Srowig

KIP, Heidelberg, Germany

M. Trieloff

University Heidelberg, Heidelberg, Germany

P. Palumbo

University Parthenope, Napoli, Italy

Springer 
stored and sealed in a MIRKA-type sample return capsule. SARIM will return the capsule containing the stardust to Earth to allow for an extraction and investigation of interstellar samples by latest laboratory technologies.

Keywords Interstellar dust - Interplanetary dust - Collection · Interstellar medium . Dust collector $\cdot$ Sample return $\cdot$ Chemical composition

\section{Science}

About 15 years ago, galactic dust traversing our planetary system was identified by the dust detector onboard the Ulysses spacecraft. Since then, analyses of data obtained at and within the Earth's orbit by different spacecraft (Helios, Galileo, and Cassini) show, that a significant amount of interstellar dust (ISD) is within our reach (Fig. 1) [1-3]. This observation opened the possibility for the Stardust mission to make the first attempt at capturing a few tens of contemporary ISD grains and delivering them to the Earth where they are subject to detailed analysis $[8,68]$.

Interstellar grains are the main repositories of condensable elements in the interstellar medium. Dust directly links the roots of our Solar System with processes of star formation and even the origin of life. However, a significant portion of cosmic dust measurements leading to new understandings has been made courtesy of remote-sensing methods. Since scientific discoveries demand confirmation and open doors to new research paths, we need alternate methods of studying cosmic dust.

The SARIM mission combines in-situ analysis of interstellar and interplanetary dust with a sample return of interstellar dust. Due to the many-fold properties of cosmic dust, dust science is an interdisciplinary research area bringing astrophys-

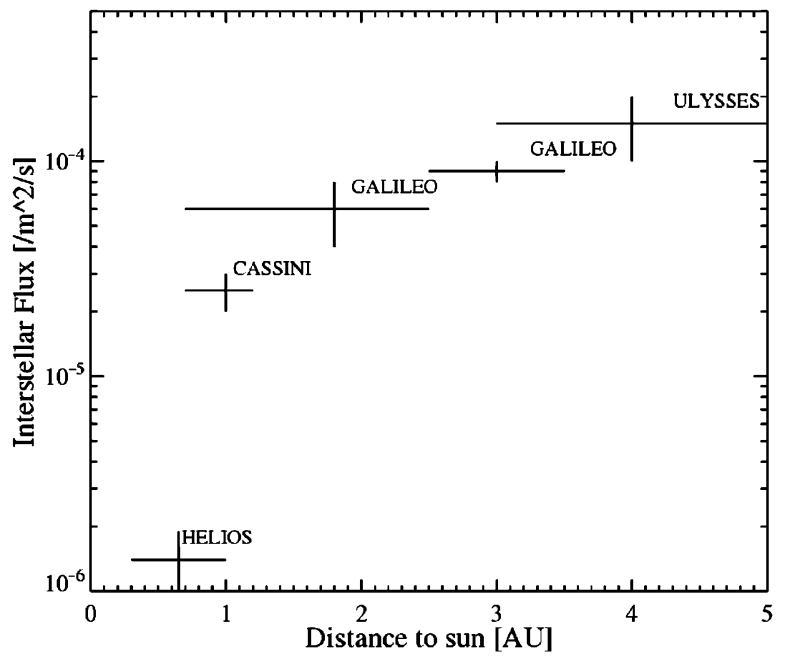

Fig. 1 Interstellar dust flux measured by in situ dust instruments in the inner solar system (from Altobelli et al. 2005a) 
icists, geophysicists, chemists, astrobiologists, and planetary scientists together. The recent progress and success in dust sensor technologies (dust telescope, dust collectors) and in dust sample returns (the Stardust mission) makes the achievement of the scientific goals now possible.

\subsection{Interstellar dust}

Interstellar dust grains are prevalent in almost every astrophysical environment, and they manifest their existence in a variety of ways. Yet, the origin and production rate of these grains is still uncertain. Interstellar dust, consisting of small submicrometresized grains of predominantly silicate and carbonaceous composition can be found in the interplanetary medium, in our Solar System, in the gaseous nebulae around stars that have completed their main-sequence lifetimes, in the interstellar medium (ISM) of galaxies like our own, in extremely luminous galaxies undergoing intense star formation or harboring a massive black hole, as well as in very young galaxies at correspondingly large distances from the Milky Way.

Even though interstellar dust constitutes less than $1 \%$ of the mass of the ISM, it plays a crucial role in regulating its thermal energy balance, in catalysing chemical reactions in molecular clouds, and in providing the basic building blocks for the formation of planetesimals in protoplanetary disks around main-sequence stars. In our Galaxy, the presence of dust grains is primarily inferred from their interaction with electromagnetic radiation, which gives rise to absorption, scattering, and polarization of Galactic starlight; infrared, submillimetre, and microwave emission, including the broad solid-state emission and absorption features; and the production of scattering halos around X-ray sources. In addition, the existence of dust is also inferred from the depletion of refractory elements from the gas phase of the ISM. Meteorites contain "old" interstellar or "presolar" dust, while SARIM is supposed to collect contemporary interstellar dust. So far it is unknown if the properties of interstellar dust have changed during the last $4.6 \mathrm{Ga}$.

Finally, interstellar dust grains have been found in meteorites on Earth, with peculiar isotopic compositions that, like cosmic fingerprints, enable scientists to trace their origins back to the dust factories that produced them.

Following their production, interstellar dust grains are injected into the diffuse ISM where they are subjected to a variety of processes. Expanding supernovae blast waves sweep up the grains, subjecting them to various destructive processes, including thermal sputtering, vaporising grain-grain collisions, and collisions that shatter large grains into smaller fragments. As the shocked gas cools, the surviving grains may find themselves inside the relative protective environment of the denser molecular clouds, where chemical reactions, accretion from the gas, and coagulating collisions reconstitute the grains, permeating and coating them with complex refractory organic compounds and more volatile ices.

We have an incomplete understanding of the efficiency of the physical processes that form, destroy, or reconstitute the grains in the ISM. The main interstellar dust factories are Asymptotic Giant Branch (AGB) stars, which produce dust in quiescent mass outflows, and core-collapse supernovae, which return their nucleosynthetic products explosively back to the ISM. A single supernova can then produce about one solar mass of dust. As grains condense in 
the cooling supernova ejecta, they preserve the distinct isotopic composition of the elements that precipitated from the gas.

Observations at infrared, radio, millimetre, and submillimetre frequencies demonstrate that a large variety of organic molecules are present in the dense interstellar gas [15]. These include organics such as nitriles, aldehydes, alcohols, acids, ethers, ketones, amines, and amides, as well as long-chain hydrocarbons. The effective shielding of UV radiation by dust in such high-density regions enables complex gas-grain chemistry in the so-called "hot-core" regions close to the forming star, which allows the formation of many organic molecules, including precursor molecules for life [46].

Laboratory isotopic analysis of meteorites and interplanetary dust collected in the Earth's stratosphere has revealed the presence of presolar grains with isotopic compositions as expected in evolved stars. Primitive meteorites and interplanetary dust particles also contain complex organics, at least some of which show isotopic evidence that they have an interstellar heritage. Comets and asteroids also show spectral signatures of organics. In particular, the molecular composition of cometary ices has been found to closely resemble that of dense interstellar clouds. The direct link between stardust and the Solar System, therefore, suggests that the early Solar System was chemically enriched by both stellar ejecta and the products of interstellar processing.

Silicate and carbon-based micron-sized dust particles, that are produced in the outflows of late-type stars, provide a catalytic surface for a variety of reactions when they are dispersed in molecular clouds [21]. In cold clouds, such dust particles adsorb ice mantles of water, $\mathrm{CO}_{2}, \mathrm{CO}$, and $\mathrm{CH}_{3} \mathrm{OH}$, with smaller admixtures of $\mathrm{CH}_{4}$, $\mathrm{NH}_{3}, \mathrm{H}_{2} \mathrm{CO}$, and $\mathrm{HCOOH}$ (Fig. 2). Acetylene $\left(\mathrm{C}_{2} \mathrm{H}_{2}\right)$ appears to be the precursor for the synthesis of hexagonal aromatic rings of carbon atoms. Benzene detection has been claimed in the Infrared Space Observatory spectrum of the circumstellar envelope CRL 618 [14]. Benzene chemistry is the first step in the formation of polycyclic aromatic hydrocarbons (PAHs), fullerene-type material, and larger aromatic macromolecular material $[25,16]$. Those materials are a major fraction of the carbon in space.

Fig. 2 Model for an interstellar dust particle with a silicate core and an organic mantle

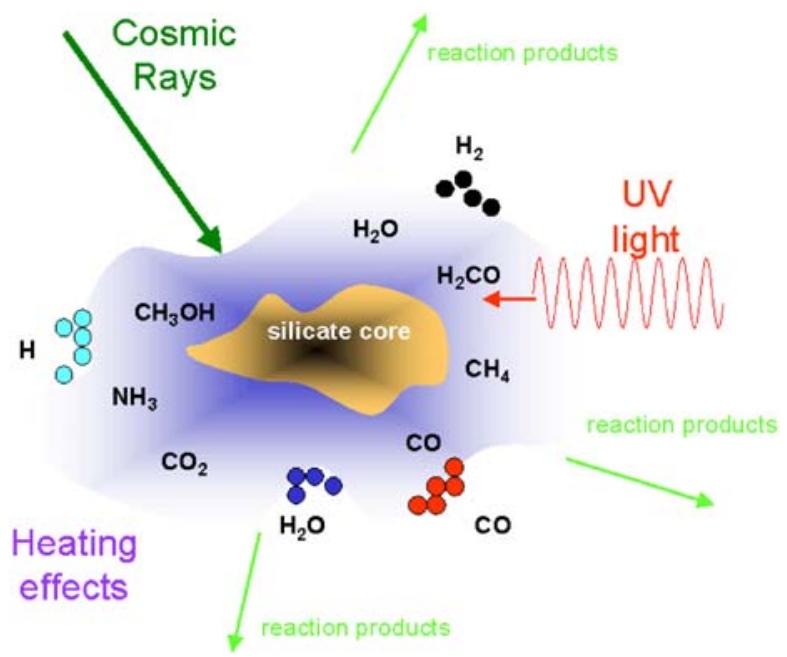


Interstellar carbon compounds and PAHs are observed in galactic and extragalactic regions. All three isoforms of carbon - diamond, graphite, and fullerene - have been identified in space environments [13] Diamonds were proposed to be the carriers of the 3.4 and $3.5 \mu \mathrm{m}$ emission bands [34] observed in planetary nebulae and laboratory infrared spectra of nanodiamonds fit well with astrophysical observations.

Graphite has not been unambiguously identified in the ISM, but is present in low abundances in meteorites $[4,54]$. The third isoform of carbon is the polyhedral C60 fullerene geometry first discussed by [45]. Fingerprints of the C60 ion were discovered in the near-infrared spectra of starlight passing through the material of diffuse interstellar clouds $[23,24]$ and higher-ordered fullerenes have been identified in meteorites [5].

SARIM will address the following questions related to interstellar dust:

- Can we distinguish original stellar condensates from dust grains that have been homogenized through processing in the ISM?

- What is the elemental composition and their isotopic signatures of grains and its variability?

- What is the nature of carbonaceous dust?

- What is the composition of silicate grains? Can we identify SiC in ISD particles and where is the iron?

- What are the sizes of compositionally different grains? Do we find nano-diamonds?

- Can destruction and formation processes (coagulation, condensation) be identified in the composition and size-distribution of ISD?

- Are there multi-component grains? Are silicates mixed-in with carbonaceous material?

- What is the shape and density of interstellar dust? Can we confirm the coremantle model of the grains? Do we find fluffy particles? What are the optical properties of the grains?

- What is the size distribution of dust at 1 AU? What is the flow direction and how does it vary with particle size and time?

\subsection{Interplanetary dust}

Interplanetary dust particles (IDPs) are more abundant than interstellar dust (ISD) in our inner Solar System. Therefore SARIM will measure IDPs alongside ISD and a major result of the SARIM mission is to learn to distinguish and characterise individual grains by their dynamical and elemental properties.

The formation of planetary bodies starts in the viscous evolution stage of the protoplanetary disc. Micron-sized dust particles initially embedded in the dilute solar nebula gas, rapidly grow (by collisional sticking) into kilometer-size planetesimals. As soon as the planetesimals are large enough to decouple from the gas motion, they move on Keplerian orbits around the protostar. Collisions between planetesimals result in the growth of larger bodies, thus producing a planet. Knowledge about the initial dust size distribution as well as the initial chemical composition of the dust would undoubtedly allow a deep insight into the general process of planet formation.

During the formation of the protoplanetary system, dust particles underwent significant alteration. Indeed, the accretion shock front produced as the molecular 
cloud collapsed would have heated and vaporised much of the dust present in the presolar nebula. The subsequent recondensation of this material explains the common isotopic composition almost everywhere in the Solar System. However, dust in the outer part of the protoplanetary disc will have been heated less, and there is certainly evidence for interstellar dust in present day Solar System material: presolar grains in meteorites, in comets, and in IDPs. On the other hand, analysis of dust samples of comet Wild 2 collected by the Stardust mission [9] showed that they are predominantly material from our solar nebula - not preserved presolar material [isotopically normal O, 51].

Cometary dust samples have a large range of varying olivine and pyroxene compositions, many particles have high abundances of $\mathrm{Mn}, \mathrm{Ca}$, and $\mathrm{Al}$ [72]. Wild 2 has a significant proportion of silicate grains that are high temperature minerals that appear to have formed in the inner regions of the solar nebula. Their presence in a comet proves that radial mixing processes were active in the early solar system.

A basic result of the temperature profile in the low pressure solar nebula is a condensation sequence for gases of molecular substances to condense directly to form solids. Condensation of a particular species occurs when the partial pressure of that species exceeds the saturation vapour pressure. Since the latter is steeply dependent on temperature, this criterion translates into a distance from the disk centre at which the species is saturated. Even though the presence of complex disequilibrium compounds and both refractory and volatile grains of presolar origin implies that the basic equilibrium condensation models are probably too simplistic [55], we can proceed with the concept that the solar nebula was likely hot enough to vaporise all but the most refractory materials within a few AU of the Sun [36], and that the composition of the parent bodies of comets, asteroids, and meteorites have gross characteristics which correlate well with the heliocentric distances at which they formed. Links between early Solar System conditions and the compositions of comets, asteroids and meteorites today (inferred from in situ and remote observations) can constrain our theoretical models of the formation of Solar System solid bodies.

In the earliest planet formation phases, whenever shocks occurred, dust particles underwent significant alteration by heating and vaporisation and recondensation. The subsequent recondensation of the presolar nebula material explains common isotopic characteristics present throughout the Solar System. While dust in the outer part of the protoplanetary disk would have been heated less, explaining some of the interstellar dust found embedded today in larger Solar System material such as meteorites, there is evidence of radial mixing in the solar nebula.

Comets, which formed in the outer Solar System, are the least altered objects surviving from the formation of the Solar System. They can preserve some record of the processes involved in their formation, and unprocessed material from the presolar molecular cloud [53]. In situ elemental and isotopic data for small newly ejected cometary particles was gathered by the dust impact time-of-flight mass spectrometers at comets Halley and Wild 2, onboard the spacecraft VeGa 1, VeGa 2, Giotto, and Stardust, respectively. It was found that the abundances of elements that are more refractory than oxygen appear to reflect solar composition, as is found for $\mathrm{C} 1$ chondrite meteorites. Particles rich in $\mathrm{H}, \mathrm{C}$, and $\mathrm{N}$ however, are less depleted in these elements than in $\mathrm{C} 1$ chondrites, and hence must be more primitive. Silicates form the most significant part of the refractory component in comets. Analysis of dust from comet Wild 2 by the Stardust mission [8, 51, 72] demonstrates that 
cometary silicates are a mix of crystalline grains (enstatite and forsterite) and glassy amorphous grains. The high temperature crystalline grains (calcium-aluminiuminclusions, or CAIs) must have been formed in the inner solar nebula and subsequently transported (radial mixing) to the comet formation zone or they may be true stardust formed in the atmosphere of another star. The amorphous grains however, appear to constitute a major fraction of non-crystalline silicates of interstellar origin.

Further evidence for relatively unaltered interstellar material being released from comets, can be gathered from the collection of fluffy stratospheric IDPs. GEMS (Glass with Embedded Metal and Sulfides) are tiny submicrometre spheroids with bulk compositions that are approximately chondritic. They form the building blocks of anhydrous IDPs in general, and cometary IDPs, in particular. Their compositions, minerology and petrography appear to have been shaped by exposure to ionising radiation. Since the exposure occurred prior to the accretion of cometary IDPs, and therefore comets themselves, GEMS are likely to be either solar nebula or presolar interstellar grains. Moreover, the properties of GEMS (size, shape, mineralogy) bear a strong resemblance to those of interstellar silicate grains as inferred from astronomical observations [7]. In addition, IDP isotopic anomalies of $\mathrm{H}, \mathrm{N}, \mathrm{O}$ indicate a presolar or interstellar origin and, in some IDPs, hotspots of extreme isotopic anomalies up to a factor of 50 have been found [52].

Dust particles that carry organic components possibly provided the building blocks for eventual life on Earth. Recently, cometary dust has been discovered to be rich in organics $[43,44]$. Carbonaceous meteorites contain a substantial amount of carbon (up to $3 \%$ by weight) and exhibit evidence of many organic molecules [57]. Therefore any collisions with such objects would have increased the population of such molecules in the Solar System, lending support for similar processes elsewhere in the Galaxy.

Cosmic dust is ubiquitous in our Solar System and easily seen in cometary comae and tails, in planetary rings, on asteroidal regolith, storms on Mars, in the Earth's stratosphere, embedded in polar ices, and dispersed in the interplanetary dust cloud (for a recent review of interplanetary dust, see [32]. As interplanetary dust lives a short lifetime compared to the life of the Sun, and is the most processed (being formed, destroyed, and/or locked into a frozen state) of the different populations of cosmic dust, in order to understand the steps of our Solar System evolution, we must accumulate dust data at different stages of its life. As interplanetary dust reveals the properties of its parent, we can form a complete 'movie' of the Solar System's evolutionary steps. The interplanetary dust complex is continually replenished by the release of dust from a variety of sources, the most visible being comets. Sublimation of volatile ices in the comet's nucleus releases dust into space, forming the comet's coma and tail, and releasing particles in trails. Fresh cometary trails as well as old cometary dust become part of the interplanetary dust cloud. To understand fresh comet dust, in-situ dust experiments measured the size distribution of dust from submicron to millimetre size in the inner coma of comet Halley [49]. The results demonstrated that large grains (mass $>10^{-6} \mathrm{~g}$ ) were more numerous than was previously expected, which dominates the mass output of the comet. This behaviour, a relatively shallow mass distribution at large grain masses, was seen again at Stardust's encounter with comet Wild $2[28,35]$. 
Another important source of interplanetary dust is derived from collisions in the asteroid and Kuiper belts. Impacts onto the surfaces of asteroids and Kuiper belt objects, and catastrophic collisions within the belts, generate fragments covering a wide size distribution of dust particles. For example, dust bands associated with the asteroid belt, which were identified using IRAS [20], demonstrated an increased dust spatial density. Since the composition of asteroid surfaces reveal processed compact silicate or metal-rich material, the interplanetary dust compositions should reflect the larger body's surface. However, the recovery of carbonaceous chondrite material from fallen meteorites indicates that there are also relatively primitive compositions to be sampled, particularly in the Near Earth Object population.

After decades of study of the interplanetary cloud, the visible zodiacal light, comets, and asteroids, and after 40 years of flying dust flux sensors in space, the relative contribution to the interplanetary dust cloud from asteroids and comets is still unknown. By combining remote dust observations with data from in-situ dust detectors, and laboratory analysis of impact craters on the surfaces of lunar rocks and spacecraft surfaces, we have a reasonably good understanding of the time averaged dust flux at $1 \mathrm{AU}$ [29]. The key to solving the 'asteroidal versus cometary' problem lies in sampling the interplanetary dust in-situ, and being able to combine accurately determined orbits with compositional data, so being able to distinguish between the sources of the dust.

Scientific questions related to interplanetary dust are

- What is the ratio of cometary versus asteroidal particles at 1 AU?

- How much is dust from comets chemically different to dust from asteroids?

- What are the orbital characteristics of different types of cometary and asteroidal particles at 1 AU?

- How complete was the chemical, isotopic, and mineralogical homogenisation in the proto-planetary nebula?

- Is today's interstellar material different from the ancient 4.6 Ga old material incorporated in comets and asteroids? Which part of presolar material is in interplanetary dust?

- What are the dust charging mechanisms?

- Are there large organic molecules in cometary and asteroidal dust that can be precursors of life?

\section{Mission profile}

In order to collect and measure interstellar dust in the inner Solar System, the interstellar particles have to penetrate the heliosphere to reach distances as close as 1 AU. The motion of interstellar grains through the Solar System is parallel to the flow of neutral interstellar hydrogen and helium gas. However, many grains are deflected by radiation pressure forces due to their optical properties (Fig. 3). Nevertheless interstellar dust was identified by in situ measurements as close as 0.3 AU from the sun [2], (Fig. 1)! The radii of clearly identified grains range from 0.1 to $1 \mu \mathrm{m}$ with a maximum at about $0.3 \mu \mathrm{m}$. 


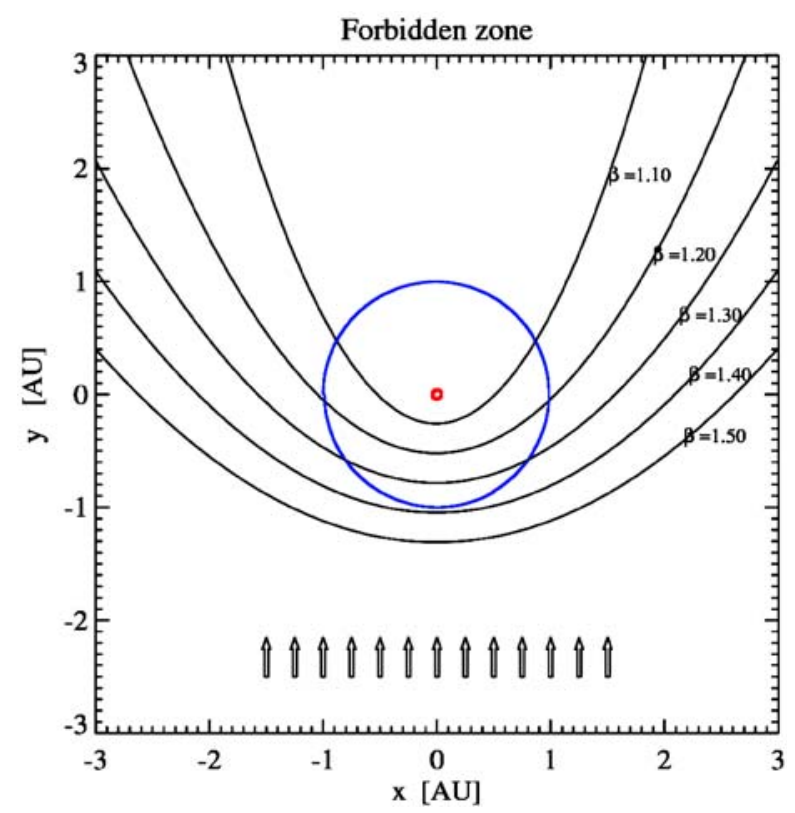

Fig. 3 Penetration depth (curves) of the interstellar dust stream (arrows). The ratio of radiation pressure to gravity determines the penetration depth, grains with ratios larger than 1.4 are effectively deflected by the solar radiation pressure and do not reach the Earth' orbit. Grains with dominating gravity are focused behind the Sun (not shown)

The SARIM spacecraft will be launched into an orbit around the Sun-Earth Lagrange point L2 which is 1.5 million km away from Earth outwards. The scientific instruments collect and measure ISD during a three year mission to meet the objectives before dust samples will be returned to Earth within a small reentry capsule. A low delta-v and low complexity strategy was chosen for the transfer to L2 and return to Earth trajectory minimising systems and operations costs.

Europe's future small low-cost launch vehicle VEGA offers $2,300 \mathrm{~kg}$ into a $5.2^{\circ}$ inclination low Earth orbit and provides enough performance to deliver SARIM as a primary payload of $2100 \mathrm{~kg}$ total launch mass into an intermediate low Earth orbit. The $480 \mathrm{~kg}$ SARIM spacecraft (including the $130 \mathrm{~kg}$ return capsule) uses an additional hydrazine-based propulsion module of $225 \mathrm{~kg}$ dry mass for transfer orbit injection. The propulsion module containing $1395 \mathrm{~kg}$ propellant provides the necessary delta-v of $3,65 \mathrm{~m} / \mathrm{s}$ for a single-burn manoeuvre into transfer orbit to L2 (Fig. 4). After separation from the propulsion module the transfer of the SARIM spacecraft takes approximately 75 days followed by direct propellant-less insertion into the Halo orbit of 205,000 km x 650,000 km around L2. All instruments of the three-axis stabilised spacecraft are fixed and provide a data volume of approx. $150 \mathrm{Mb}$ per day which is downlinked every 2 weeks.

The total number of collected interstellar and interplanetary particles is dependent on the relative dust impact speed which is modulated over one year. Assuming an apex pointing of the collectors, the interplanetary flux is expected to be constant with time whereas the interstellar dust flux is highly variable [33]. The relative impact speed of interstellar grains with beta equal one (radiation pressure and gravity are 

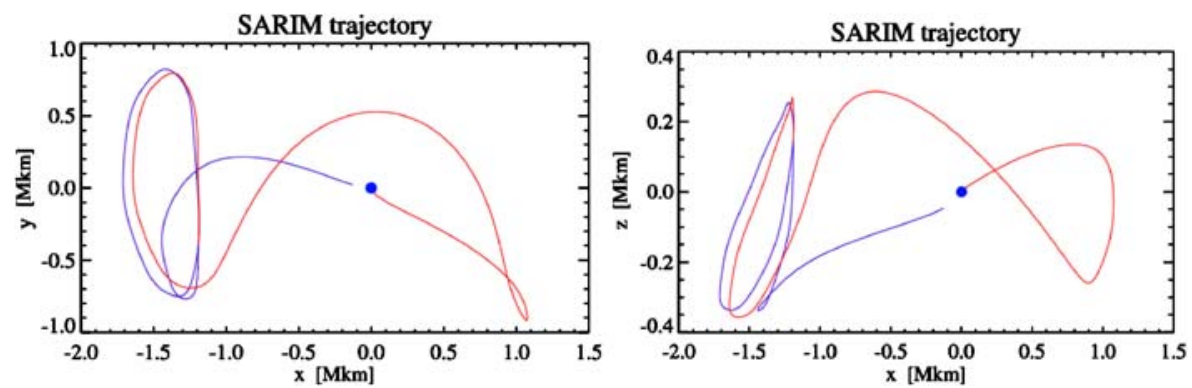

Fig. 4 SARIM trajectory in a barycentre fixed rotating system in the ecliptic plane (left). Transfer trajectory from low Earth orbit and direct insertion into Halo orbit around L2 (blue). Free return trajectory (red) from L2 of SARIM during its 7 months cruise phase to transport the dust sample re-entry capsule back to Earth

equal) varies between zero and $57 \mathrm{~km} \mathrm{~s}^{-1}$ (Fig. 5). In this scenario it is expected that 100 interstellar dust grains are collected with a $1 \mathrm{~m}^{2}$ sensor area per year with moderate impact speeds of below $20 \mathrm{~km} \mathrm{~s}^{-1}$ which keeps the dust grains almost intact after impact (Fig. 6). A detailed operations scenario has to be developed by the scientists after the selection of the various capture materials. The scenario shall guarantee the highest cleanliness of the collector media and a good contrast between interplanetary and interstellar dust capture. For this purpose the collector material shall be exchanged at least twice per year. The collection strategy should also take into account the varying relative impact speed of the interstellar dust over time. The detailed collection scenario is not relevant for the overall mission profile, since the spacecraft is three-axis stabilised and data of the in-situ instrumentation are transferred once every 2 weeks. More than $90 \%$ of the time the spacecraft can be pointed according to the scientific needs (towards the interstellar dust RAM direction but avoiding the Sun in the dust telescope apertures).

After three years of collection and in-situ measurements around L2, the SARIM spacecraft with the return capsule attached performs an Earth return transfer manoeuvre demanding a delta-v of less than $15 \mathrm{~m} \mathrm{~s}^{-1}$. After a 7 months journey the

Fig. 5 Relative impact speed of interstellar dust particles at an Earth-like orbit with different beta values

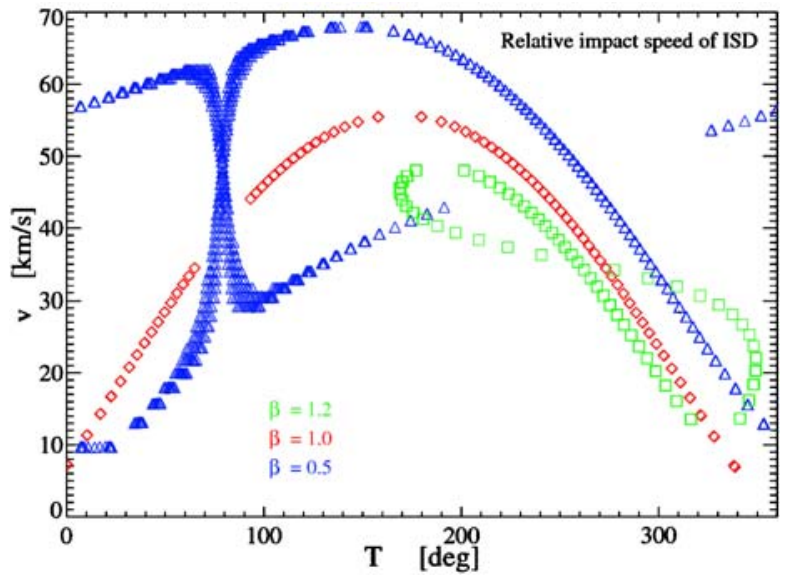




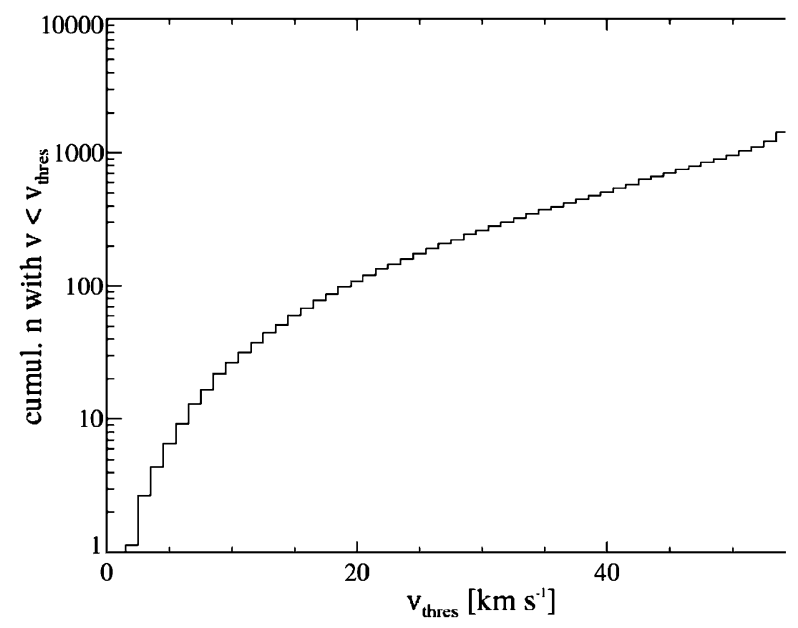

Fig. 6 Cumulative flux of interstellar dust grains above a certain speed threshold. SARIM will collect 1,000 interstellar grains within 1 year of all relative impact speeds. Approx. 100 grains are collected with impact speeds below $20 \mathrm{~km} \mathrm{~s}^{-1}$

return capsule is released from the SARIM bus approximately $1 \mathrm{~h}$ before Earth reentry for precise and safe guidance. The spacecraft is transferred into a heliocentric orbit with a rescue manoeuvre of $57 \mathrm{~m} \mathrm{~s}^{-1}$ delta-v after capsule separation for possible mission extension.

The ROM costs of the spacecraft bus (excluding payload) are approx. $125 \mathrm{M} €$ including the sample return mechanism and capsule; the preferred launcher of type VEGA require an additional budget of $20 \mathrm{M} €$.

\section{Payload concept}

The in-situ instruments will analyse the dust flux, size distribution, dust trajectories (dynamics and origin), and coarse elemental composition. They will also study time variability of the interstellar dust stream through the heliosphere and its coupling to the solar cycle. The preliminary payload consists of seven Active dust Collectors (AC), one Dust Telescope (DT), one Nano-particle detector, a plasma monitor, and one Sample Return Capsule (SRC). This payload allows a sensitive and reliable determination of individual dust grain characteristics such as speed, mass, charge, trajectory and composition. The total science payload mass is $86 \mathrm{~kg}$ and requires $170 \mathrm{~W}$ (Table 1).

\subsection{Active dust collectors}

Seven Active Collectors (AC) are used onboard SARIM to monitor the dust flux with a total sensitive area of $1.1 \mathrm{~m}_{2}$. These sensors are capable of determining the impact time, velocity vector, trajectory (hence grain origin), primary charge, impact location at the collector surface and mass of individual micrometeoroids. Each AC consists of a Dust Trajectory Sensor (DTS), a movable dust collector unit, and an 
Table 1 SARIM model payload summary table

\begin{tabular}{|c|c|c|c|c|c|c|}
\hline Payload & $\begin{array}{l}\text { Number } \\
\text { of } \\
\text { modules }\end{array}$ & $\begin{array}{l}\text { Mass } \\
\text { per } \\
\text { module }\end{array}$ & $\begin{array}{l}\text { Power } \\
\text { per } \\
\text { module }\end{array}$ & $\begin{array}{l}\text { Data } \\
\text { volume } \\
\text { per day } \\
\text { (MB) }\end{array}$ & $\begin{array}{l}\text { Dimensions } \\
\left(\mathrm{cm}_{3}\right)\end{array}$ & Dust measurement \\
\hline $\begin{array}{l}\text { Dust trajectory } \\
\text { sensor }\end{array}$ & 7 & $6 \mathrm{~kg}$ & 13 & 16.2 & $40 \times 40 \times 20$ & Speed, charge, mass, trajectory \\
\hline Active collector & 7 & & & & & \\
\hline Dust collector & 7 & $0.5 \mathrm{~kg}$ & 0 & - & $38 \times 38 \times 1$ & Composition \\
\hline Dust camera 1 & 1 & 4.9 & 8 & 1 & $30 \times 30 \times 34$ & Flux, dust crater \\
\hline Dust camera 2 & 1 & 5.6 & 36 & 1 & $36 \times 36 \times 29$ & Flux, dust momentum \\
\hline Dust camera 3 & 5 & 2.5 & 2.0 & 1 & $40 \times 40 \times 3$ & Flux, mass, Energy \\
\hline Dust telescope & 1 & 25.0 & 20 & 21.6 & $\mathrm{D} 650 \times 720$ & $\begin{array}{l}\text { Flux, speed, trajectory, mass, charge, } \\
\text { composition }\end{array}$ \\
\hline Plasmon & 1 & 1.3 & 1.5 & 1.3 & $15 \times 15 \times 15$ & Electrons, ions, mag. field \\
\hline AFIDD & 1 & 1.0 & 2.0 & 1 & $13 \times 13 \times 2$ & Flux nano-dust \\
\hline Total & & 85.8 & 168.5 & 140 & & \\
\hline
\end{tabular}

impact detector (ID) (Fig. 7). The combination of DTS with ID is also called a Dust Camera (DC). A robotic mechanism pushes the collector between the DTS and the ID for the exposure to interstellar grains during parts of the orbit (i.e. July to October). For non-exposure times the collector is removed and the dust grains pass through the DTS and impact onto the ID, which itself is a separate dust sensor applying a variety of detection techniques to get complementary information about the dust grains. Detection techniques of these impact detectors are either impact ionisation (used by five modules), depolarisation of foils (one module), or acoustic signals (one module).

A single DTS module consists of four sensor grids mounted between two electrical shielding grids (Fig. 8). The distance between grid planes is $40 \mathrm{~mm}$. Each sensor grid consists of 15 parallel wire electrodes and the wires within one plane are separated by $20 \mathrm{~mm}$. Each electrode has a capacitance of about $5 \mathrm{pF}$ and is connected to a separate charge sensitive amplifier (CSA) with a very low noise of 100 electrons. Dust particles' trajectories are determined by the measurement of the electric signals that are induced when a charged grain passes through the position

Fig. 7 Cut through an active dust collector consisting of a Trajectory Sensor (top), a dust collector material (green, middle) and an impact detector (bottom, brown)

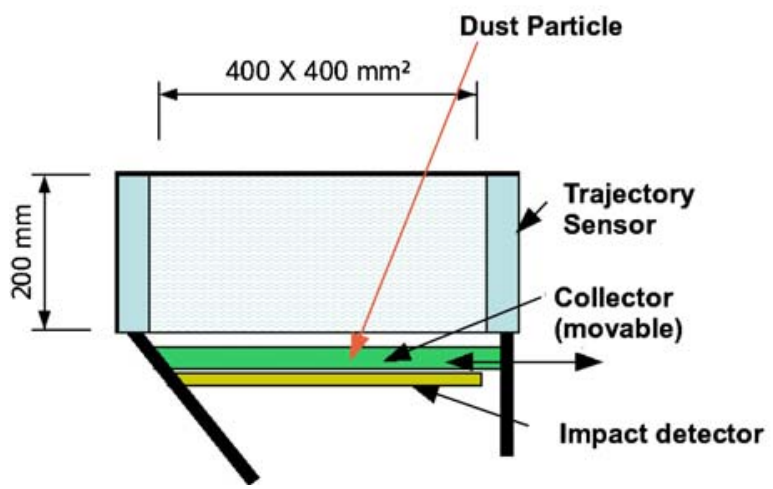


Fig. 8 Cut through the Dust Trajectory Sensor. Two shielding grids and four planes of parallel wires provide (via charge induction) the dust particle trajectory

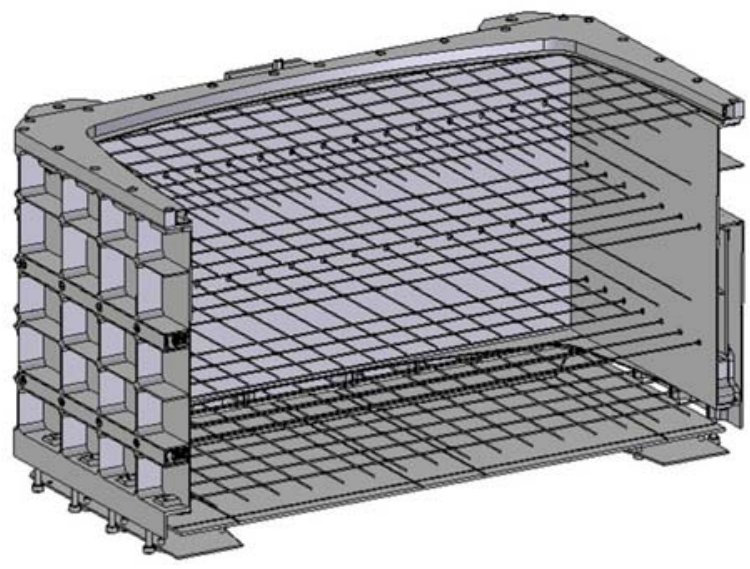

sensitive electrode system. The objective of the trajectory sensor is to measure dust charges in the range $10^{-16}$ to $10^{-13} \mathrm{C}$ and dust speeds in the range 6 to $100 \mathrm{~km} \mathrm{~s}^{-1}$. Laboratory tests have demonstrated excellent performance. An ASIC charge sensitive amplifier and an ASIC transient recorder have been developed with a RMS noise of about $1.510^{-17} \mathrm{C}$. The velocity vector of individual dust grains will be determined with an accuracy of better than $3^{\circ}$ and a magnitude of better than $3 \%$. The Cosmic Dust Analyser onboard Cassini was the first dust detector measuring the primary charge of individual dust grains in space [42].

For deep-space collection of interplanetary and interstellar particles (dominated by silicate/sulfide/oxide minerals with very small grains including amorphous, non-crystalline materials, diamond, exotic carbides and nitrides), the ideal collector substrate must not contain elements likely to be of major significance in captured particles ( $\mathrm{Na}, \mathrm{Mg}, \mathrm{Al}, \mathrm{Si}, \mathrm{P}, \mathrm{S}, \mathrm{K}, \mathrm{Ca}, \mathrm{Ti}, \mathrm{Cr}, \mathrm{Mn}, \mathrm{Fe}, \mathrm{Ni}) . \mathrm{H}, \mathrm{C}, \mathrm{N}$, and $\mathrm{O}$ should be absent, or their isotopic composition in the substrate should be highly distinctive if they are to be distinguished from captured organic material. Furthermore, the collection medium should: capture any particle between $10 \mathrm{~nm}$ and $1 \mathrm{~mm}$ size, regardless of particle structure, mass or velocity; result in no structural or compositional modification during or subsequent to capture; allow rapid and unambiguous location of all captured particles on return to the laboratory; give no interference with in-situ analysis techniques, and no ambiguity as to the origin of specific elemental signatures; permit perfect physical separation of the captured particle prior to further, sophisticated analysis. At present no material meets all of the desirable criteria perfectly. However, three probably provide the best available compromise: silica aerogel, polymer, and metal foil surfaces, albeit in modified form to those deployed on previous missions, and each with different limitations.

Silica aerogel as collector substrate material is very low in density (as low as $0.002 \mathrm{~g} \mathrm{~cm}^{-3}$ ) and highly porous. Impactors can tunnel with varying degrees of damage depending on the particle structure, composition, size and speed $[10,71]$. Dense and robust particles penetrate deeply, and little material is lost or dust structure modified. The peak pressure at $6 \mathrm{~km} \mathrm{~s}^{-1}$ is likely to be only a few GPa. 
Coarser grained silicates, oxides, and carbonates are known to survive relatively intact, suitable for both mineralogical and petrological analyses and determination of major, minor and trace elemental composition as well as isotopic characterisation.

Recent studies focus on the development of non-silicate aerogels to allow for a detection of silicates in the collected dust grains. Non-silicate materials studied are of type carbon, alumina, titania, gemrania, zirconia, niobia, tin oxides and hafnia with densities between 20 and $100 \mathrm{mg} / \mathrm{cc}$. Although such materials are opaque, synchrotron X-ray microprobe studies (SXRM) allow the measurement of elemental abundances. It was found by hyper-velocity impact tests at the Ames Research Center that carbon based aerogel out of resorcinol-formaldehyde (RF) are well suited to locate, extract and investigate micro-meteoroids with standard laboratory techniques [37].

Polymer foil multilayer blankets are relatively thin layers of approx. $1 \mathrm{~g} \mathrm{~cm}^{-3}$ density, held with open space between. They permit penetration and a degree of particle disruption without significant loss due to ejection, allowing capture of fine fragments and residues [38, 39]. In situ analysis is straightforward as grains and residues are on the foil surface [27]. High vacuum electron- and ion-beam methods can be used if there is a very thin metallic coating. The compositional signature of the impactor, other than for organic particles, is easy to distinguish from the substrate.

Metal plates and foils as dense targets have been widely studied and their cratering characteristics have been intensively interpreted as a function of particle size, speed, structure, density, and impact residue composition, using a very diverse range of instruments [6, 40, 41, 47]. Recent studies have shown that almost all craters contain residue, and can provide information about particle internal structure. At $6 \mathrm{~km} \mathrm{~s}^{-1}$ a substantial proportion is retained on the floor of aluminium foil craters, probably exceeding $30 \%$ of the original mass. More material is found on the crater walls and lip, although this may have undergone greater alteration.

For a later comparison of surface contaminations, each collector material used shall be mounted at the spacecraft at different locations such that they are exposed to the spacecraft environment but are protected from any dust flux. These witness media allow for a clear identification of elemental species caused by spacecraft outgassing or other environmental effects.

\subsection{PVDF impact detector}

The combination of DTS with an impact stage is called a Dust Camera (DC). The impact stage of DC 1 uses polyvinylidene fluoride (PVDF) film to determine the mass of a dust particle passing through a Trajectory Sensor. PVDF film consists of a permanently polarized material. A particle impacting the sensor surface, makes a crater which produces a local destruction of dipoles [69]. This results in a rapid current pulse (of order $10 \mu \mathrm{s}$ ) which can be detected by relatively simple electronics. The pulse signal (charge liberated) depends on particle mass and velocity. This instrument comprises 25 discrete (in a $5 \times 5$ array) PVDF film modules, each with approximate dimensions $6 \mathrm{~cm} \times 6 \mathrm{~cm}$ (Fig. 9), mounted on an acoustically isolated plate. Each PVDF film is held by an acoustically isolating housing around its perimeter. For maximum sensitivity, thin PVDF is used $(6 \mu \mathrm{m}$ thick with $70 \mathrm{~nm}$ 
thick conducting layers at both surfaces). Using 25 modules minimizes capacitance of each sensor element, as well as aiding reliability and discrimination between impact sites. The 25 submodules of the PVDF impact stage require less than $3 \mathrm{~kg}$ and $2 \mathrm{~W}$ in total.

PVDF was developed as a dust impact sensor [e.g. 58, 59] and flown successfully on various missions [70]. A PVDF instrument was flown aboard the Stardust spacecraft [70] and is currently flying aboard Cassini as part of the Cassini Cosmic Dust Analyser, CDA [60].

\subsection{PZT impact detector}

The detection method of the 2nd impact stage (ID 2) is based on a metal diaphragm exposed to the impact of grains. The plate is equipped with piezoelectric sensors (PZT, e.g., lead zirconate titanate crystals); their number is adequate to have multiple measurements of a single impact, which give impact position and an intrinsic redundancy in the sensor. When a grain impacts the sensing plate, flexural waves generated on the plate are detected by the PZTs. The maximum displacement of these elements is related to both mass and speed of an impinging grain, being closely proportional to its momentum, and the displacement of the crystal produces a proportional potential. This effect strongly depends also on impact speed, due to variation of impact physical effects and the coefficient of restitution [22]. The impact sensor is capable of providing information about the flux of particles encountered and a combination of their mass and speed, very closely related to the momentum. If the relative velocity of the impactor with respect to the local environment is measured by the trajectory sensor, the mass of each particle can be derived.

The impact sensor plate developed for previous experiments (e.g., GIADA) has a $0.5 \mathrm{~mm}$ thick aluminium diaphragm and square sensitive area $0.01 \mathrm{~m}^{2}$. ID2 for SARIM will have a sensitive area of $0.1 \mathrm{~m}^{2}$, which can be guaranteed by integrating several parallel modules or by developing a larger module. The typical structure of the ID2 or one of its sub-modules is represented in Fig. 10, where the instrument is shown open, with the sensing plate on the right to expose 5 PZT sensors calibration unit attached to it, while the proximity electronics is below a protective plate on the left side.

The performances of several types of impact sensors have been checked in the past, both in the laboratory and in space applications. Tests have been performed with real grains with different velocities [17-19] and a minimum momentum of $6.5 \times 10^{-10} \mathrm{~kg} \mathrm{~m} \mathrm{~s}^{-1}$ can be detected. Saturation limit is expected at $4.0 \times 10^{-4} \mathrm{~kg} \mathrm{~m}$ $\mathrm{s}^{-1}$. The detection limit corresponds to mass sensitivity of $1.3 \times 10^{-13}, 6.5 \times 10^{-14}$ and $3.2 \times 10^{-14} \mathrm{~kg}$ for 5,10 , and $20 \mathrm{~km} \mathrm{~s}^{-1}$ relative speed.

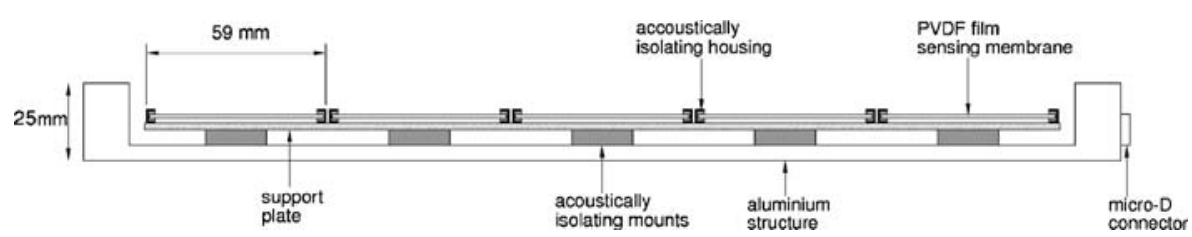

Fig. 9 Side view of the PVDF film modules for the impact stage of Dust Camera 1 


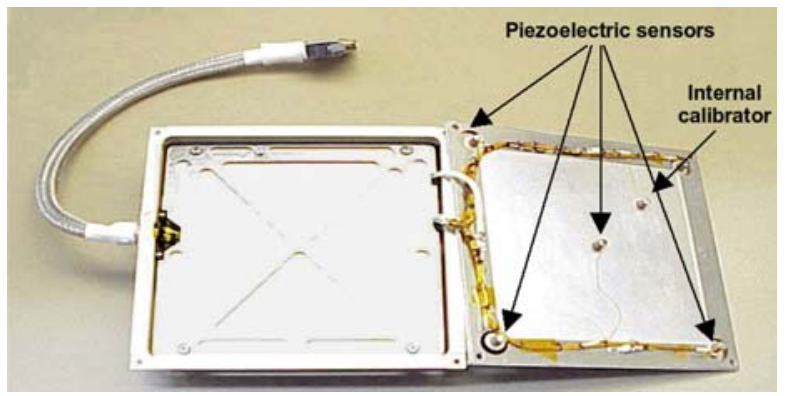

Fig. 10 Interior of an impact sensor with PZTs developed for the GIADA instrument onboard Rosetta. The PZTs are mounted on the rear of the impact plate (right). The electronics are integrated below the sensor (right)

\subsection{Ionisation impact detector}

The third Dust Camera uses an impact stage utilizing the process of impact ionization (ID 3), which occurs when a particle at hypervelocity impacts a target. In situ data from such instruments have been obtained over several decades. The sensors aboard Galileo [30], Ulysses [31] and Cassini [60] return unprecedented data.

In this instrument, a particle impacts a flat target plate (at $0 \mathrm{~V}$ ), and impact cations are accelerated towards an electrode grid (at $-100 \mathrm{~V}$ ). The total charge collection at this electrode is related to the particle mass and velocity (by $m v^{3.4}$ ). As velocity is independently determined by the DTS in front of ID 3, the mass of the particle can thus be obtained. The instrument comprises 25 sensing modules, each $9 \mathrm{~cm} \times 9 \mathrm{~cm}$, mounted in a $5 \times 5$ array similar to the PVDF-based camera. The sensing grid is shielded by a further pair of grids (at $0 \mathrm{~V}$ ). Each set of grids is held in a frame around its perimeter. This instrument contributes less than $4 \mathrm{~kg}$ and $2 \mathrm{~W}$ to the total mass/power budget of the whole dust camera.

\subsection{Dust Telescope (DT)}

The Dust Telescope combines a Trajectory Sensor with an analyser for the elemental composition of micrometeoroids [62] (Fig. 11). The integration of the two subsystems to one high-performance detector allows for a simultaneous determination of the dust particle mass, velocity vector, surface charge and composition.

The large area mass analyser is based upon impact ionisation of hyper-velocity dust impacts onto a ring shaped target plate [62, 67]. This time-of-flight system uses a reflectron for an increased mass resolution and provides the elemental composition of individual micrometeoroids with a mass resolution between 100 and 300 [61, 63]. It consists of a ring shaped target within a cylindrical housing (Fig. 11). The impact generated ions are accelerated and pass the field free region of the Trajectory Sensor until they enter a reflectron formed by a parabolic shaped grid. Finally, the ions are detected at a large ion collector producing a time-of-flight spectrum (Fig. 12). Although a similar instrument is already flown onboard Stardust, the spectrometer of the Dust Telescope has a sensitive area 10 times larger. 
Fig. 11 Cross section of the dust telescope. The dust particle impacts onto the target and the impact generated ions are focused onto the ion detector by a reflectron. A cover protects the spectrometer from contaminations during launch and in the early cruise phase

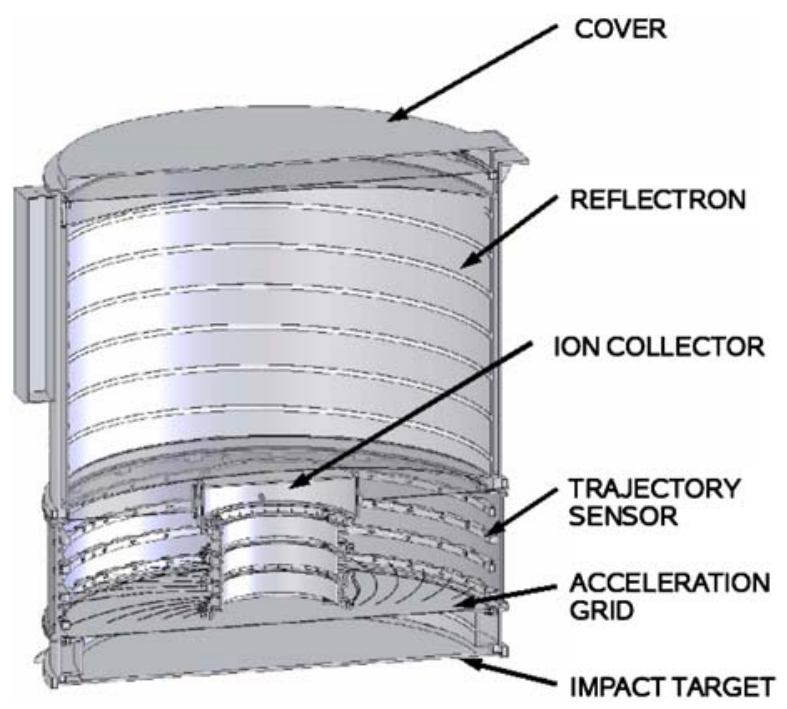

\subsection{Nano-dust detector}

The basis for the stand-alone Aluminium Film Interplanetary Dust Detector (AFIDD) is a thin (10-100 nm) Al film [see 11, 12]. Dust particles impacting on the film will have a ballistic limit defined as the maximum thickness of aluminium which can be perforated [50]. The limit is a function of both the mass and velocity of a particle and is derived from empirical formulae generated from laboratory impact data [26]. The Al films are supported by the interchannel walls of an microchannel plate (MCP) detector and are freestanding over the open area of the microchannels.

AFIDD consists of an array of four circular, $\mathrm{Al}$ filmed MCP detectors bearing two film thicknesses $(2 \times 10 \mathrm{~nm}$ and $2 \times 100 \mathrm{~nm})$ to provide two ballistic limit thresholds (Fig. 13). The MCP detectors are operated in a "low-gain" mode, whereby the voltage across the channels is low compared with a standard photon or electron

Fig. 12 Time-of-flight spectrum of an iron particle impacting with $24 \mathrm{~km} \mathrm{~s}^{-1}$. The spectrum was recorded at the Heidelberg dust accelerator facility using the laboratory model of a large area mass analyser

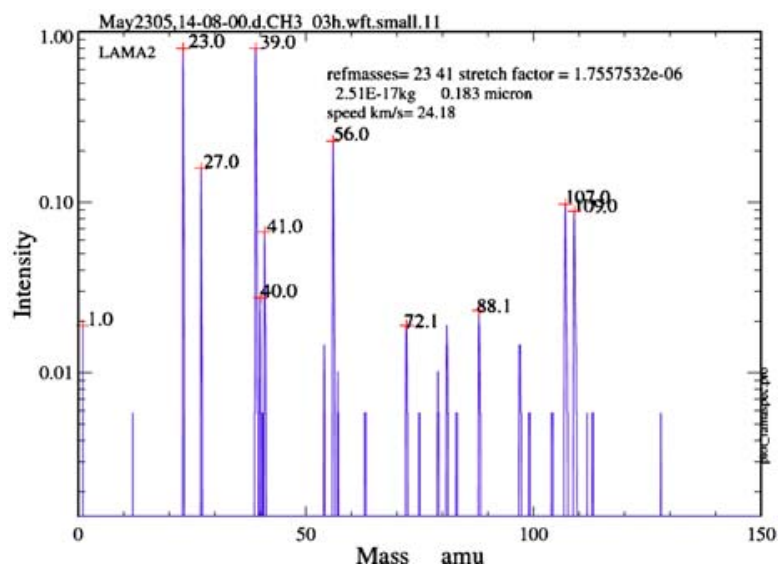


detecting MCP detector in order to prevent saturation of the electron avalanche. The result is that the pulse height becomes a function of the total energy deposited in the microchannel and can therefore be used to discriminate between dust and radiation induced events.

AFIDD will detect hypervelocity impacts by interplanetary and interstellar dust particles with masses of the order of $10^{-21} \mathrm{~kg}$ and less, at typical dust velocities. AFIDD's high sensitivity provides access to new populations of both interplanetary and interstellar dust particles, whose source bodies, dynamics and evolution differ significantly from those of larger particles. The dynamics of nanometre scale particles is dominated by the Lorenz force and it has been predicted that interplanetary nanometre scale particles in the inner Solar System, and more than a few tenths of an AU from the Sun, will be carried with the solar wind. Particles may be $\mathrm{MgO}$ from sublimation of cometary and meteoritic silicates, nanodiamonds from meteoroid material and carbon structures formed by thermal alteration of organics [48].

\subsection{Plasma monitor}

The Plasma monitor PLASMON consists of a magnetometer, an electrostatic plasma analyser and a Faraday cup (Fig. 14), which is necessary to monitor the plasma and electro-magnetic environment. All three sensors are integrated in the $300 \mathrm{~g}$ spherical PLASMON sensor. A small electrostatic plasma analyser measures the electron and ion distribution in a wide energy range. Hemispherical deflection plates are used to analyse the energy distribution in 32 increments. Assuming a radial solar wind bulk velocity, all major plasma parameters such as electron and proton densities, temperatures, and proton bulk velocities can be derived. The energy ranges measured are 40-8,000 eV (ions) and 0.35-4,200 eV (electrons). The Faraday cup measures ions up to $2 \mathrm{keV}$.

The magnetic field is measured with a vector compensated ringcore fluxgate magnetometer. The resolution of the magnetometer $(10 \mathrm{pT})$ is only restricted by the sensor noise $\left(<5 \mathrm{pT} \mathrm{Hz}^{-0.5}\right.$ at $\left.1 \mathrm{~Hz}\right)$ and fields up to $1,000 \mathrm{nT}$ can be determined. DC-magnetic fields generated by the spacecraft will be compensated internally by dedicated coils in situ.

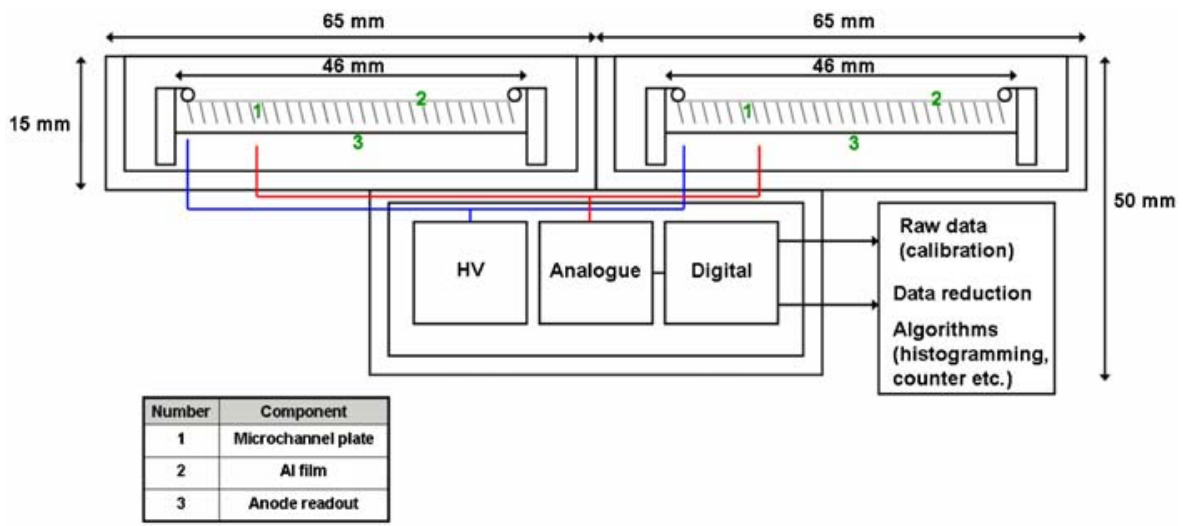

Fig. 13 AFIDD architecture 
Fig. 14 The PLASMON sensor

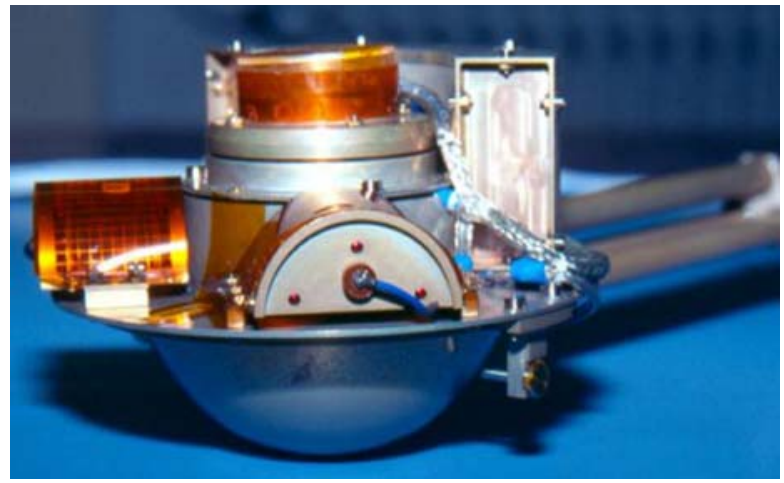

\section{Spacecraft}

A S/C design was developed by Astrium/EADS on the heritage of former missions (Fig. 15). The mission consists of one three-axis stabilised spacecraft with rotating solar cells, body mounted payload and one return capsule. The return capsule has a diameter of $1,100 \mathrm{~mm}$ and is located in the centre of the S/C bus. The collector modules are placed around the sample return capsule which contains the sample holder (Figs. 16 and 17). Seven dust collectors with a size of $400 \mathrm{~mm} \times 400 \mathrm{~mm} \times 20 \mathrm{~mm}$ and a total mass of $0.5 \mathrm{~kg}$ per collector plus supporting structure mechanism and a collector storage housing have to be accommodated within the return capsule. The return capsule cover opens only by lifting the front heatshield vertically by roughly $50 \mathrm{~mm}$ using two linear actuators (Fig. 16). The dust collectors are arranged in a stack mounted in a turn table housing within the front heat shield, which opens and thereby unseals simultaneously with the movement of the front heat shield. A

Fig. 15 SARIM spacecraft in a VEGA payload fairing with extended solar sails. Six Active Collectors (red cubes), one Dust Telescope (grey cylinder) and the collector sample stack (green) are mounted on the S/C body. The Sample Return Capsule encloses the collector samples in the centre (not shown)

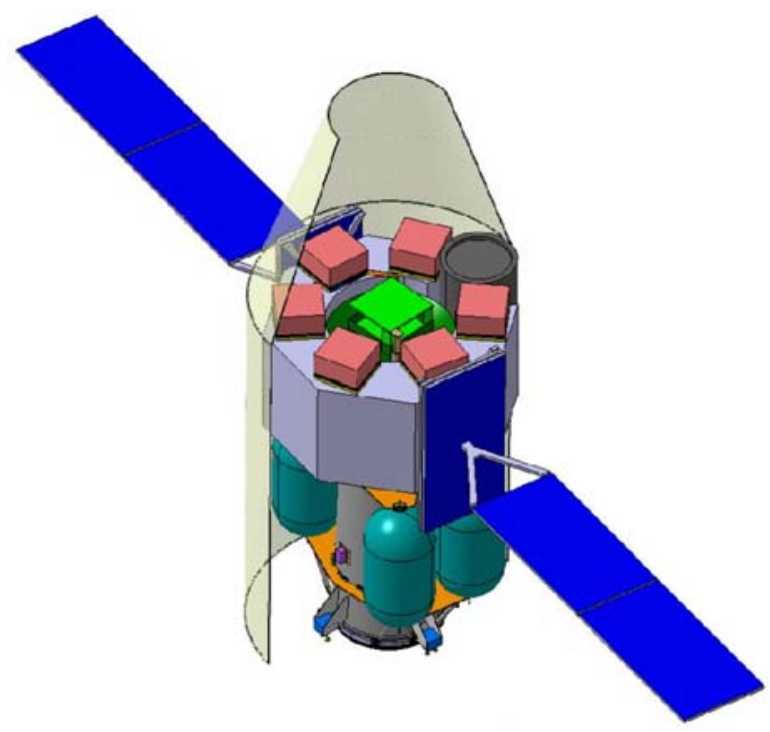


Fig. 16 SARIM payload configuration with seven collector modules, one Dust Telescope and the Sample Return Capsule in the centre

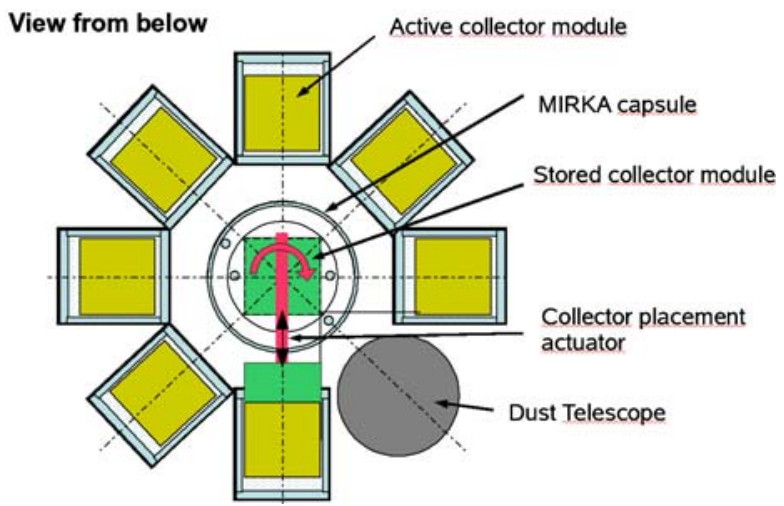

collector handling system consisting of two linear actuators for vertical movement and a "container bridge-like" sliding mechanism picks up a single collector for delivery to each of the active collectors. The MIRKA based return capsule has already demonstrated its reliability.

\section{Laboratory analysis of dust samples}

The scientific success of SARIM is related to the quality of the subsequent laboratory analysis of dust samples extracted from the collectors. Contemporary interstellar dust has never been analyzed in terrestrial laboratories so far. Therefore all kind of information that can be retrieved from returned samples is of great value and can in principle not be foreseen. First analyses of the dust grains should try to focus on four major issues: isotopic composition, inorganic chemical composition, organic chemical composition and mineralogical/petrologic composition. Each of these issues requires specific techniques and has its special demands on sample properties.

All techniques that are suited to analyze the isotopic composition of dust grains are to a certain extent destructive. For the analysis of interstellar dust, two

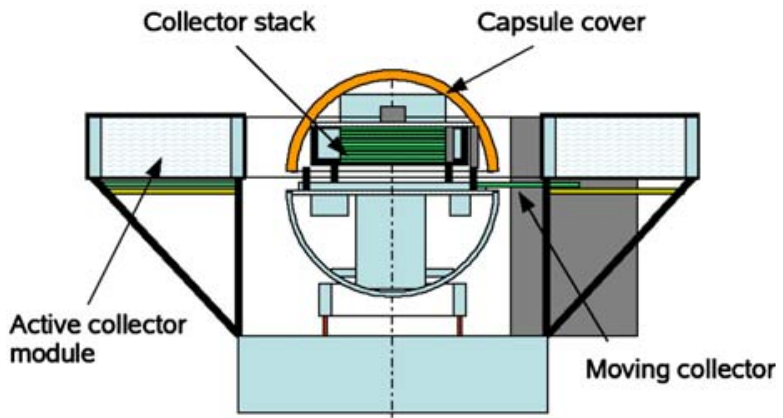

Fig. 17 SARIM payload configuration and sample collector placement. A mechanism moves collector plates (green) to the individual Active Collector Modules 
requirements are indispensable: high lateral resolution and high sensitivity. At present, the primary technique for isotope analysis of dust grains is NanoSIMS, a variant of SIMS (secondary ion mass spectrometry) instruments with high lateral resolution (50-200 nm), sufficient precision, and little sample destruction. TOFSIMS (time-of-flight secondary ion mass spectrometry) has also a high lateral resolution (200-300 $\mathrm{nm}$ ) but is less accurate. Its major advantage is that it allows analyzing complete mass spectra in a single measurement.

While from the technical point of view, isotopic analyses are certainly highly demanding, the requirements for sample collection are relatively simple. For example the only presolar dust grain that has been identified among the cometary dust collected by Stardust so far was found in a hypervelocity impact residue on aluminum foil [51, 64]. This demonstrates that even for non-intact capture of dust grains, isotopic information can be retrieved, although intact capture is certainly preferable.

For the analysis of the chemical composition of captured dust grains, further methods like scanning electron microscopes with energy dispersive spectrometers (SEM-EDS) and SXRF (synchrotron X-ray fluorescence) are available. A SEM-EDS technique was used to investigate the residues of Stardust samples that were captured on Al foil [40, 41], and TOF-SIMS results of isolated particle tracks in aerogel of collected dust were published in [56,65-67]. Here, most electron beam techniques so far failed due to intense charging of the aerogel.

In principle, to yield valuable chemical information on the collected dust grains, the requirements concerning sample collection are higher than for isotopic analysis. The chemical composition is more easily diluted or altered than the isotopic composition. Non-intact sampling always bears the risk of chemical fractionation as observed for LDEF (long-duration exposure facility) samples. Therefore, a nondestructive sampling technique is highly desirable. Contrary to the Stardust collection, at least some of the sampling material should be made from another major element than silicon. Silica aerogel, although ideal from its physical properties (low density), has the problem that $\mathrm{Si}$ is one extremely important element to be determined in these dust grains. A low-density capture medium made from a trace element that is less important to be determined in interstellar dust is therefore highly desired.

Only TEM provides the possibility to study the mineralogy and petrology of the interstellar dust particles. This investigation is important to acquire information on the exact conditions during formation of dust grains, e.g., in the outflows of stellar processes and their history in the interstellar medium. To determine their state of crystallinity is one of the principal goals of a laboratory study. It is obvious, that for mineralogical investigations only non-destructive sampling techniques can be used not affecting the structural properties.

\section{Conclusions}

SARIM is a $150 \mathrm{M} €$ class mission proposal to return interstellar and interplanetary dust to Earth. Active dust collectors with a sensitive area of $1 \mathrm{~m}^{2}$ determine the trajectory, speed, mass and impact location of individual dust impacts. This allows 
for a discrimination between interstellar and interplanetary grains for the first time. The collection and sample return technology is ready to operate SARIM for three years at the Lagrangian point L2 of the Sun-Earth system. The scientific community has grown enormously with the success of the Stardust mission and its return of cometary material to Earth in 2004. Now, the time is ripe to prepare for detailed laboratory studies of interstellar dust, which will provide the link between meteoritic analyses in the inner Solar System and astronomical observations. Although SARIM was not selected for a study in ESAs Cosmic Vision 2015-2025 program, a detailed mission study beyond the scope of this paper is highly recommended.

Open Access This article is distributed under the terms of the Creative Commons Attribution Noncommercial License which permits any noncommercial use, distribution, and reproduction in any medium, provided the original author(s) and source are credited.

\section{References}

1. Altobelli, N., et al.: Cassini between Venus and Earth: detection of interstellar dust. J. Geophys. Res. 108, (A10), 8032 (2003)

2. Altobelli, N., et al.: A new look into the Helios dust experiment data: presence of interstellar dust inside the Earth orbit. Astron. Astrophys. 448, (1), 243-252 (2005a)

3. Altobelli, N., et al.: Interstellar dust flux measurements by the Galileo dust instrument between the orbit of Venus and Mars. J. Geophys. Res. 110, (A7), A07102.1-A07102.13 (2005b)

4. Amari, S., Lewis, R.S., Anders, E.: Interstellar grains in meteorites: III. Graphite and its noble gases. Geochim. Cosmochim. Acta. 59, 1411-1426 (1995)

5. Becker, L., Bunch, T.E.: Fullerenes, fulleranes and PAHs in the Allende meteorite. Meteoritics. 32, 479-487 (1997)

6. Bernhard, R.P. et al.: Projectile compositions and modal frequencies on the "Chemistry of micrometeoroids" LDEF experiment. In LDEF, 69 months in space: First postretrieval symposium, edited by Levine A. S. Washington, D.C.: NASA, Office of Management, Scientific and Technical Information Program, 551-573 (1993)

7. Bradley, J.P., et al.: An infrared spectral match between GEMS and interstellar grains. Science 285, 1716-1718 (1999), +cover

8. Brownlee, D.E., et al.: Stardust: comet and interstellar dust sample return mission. J. Geophys Res. 108, (E10), SRD1-1 (2003)

9. Brownlee, D.E., et al.: Comet Wild 2 under a microscope. Science 314, 1711-1716 (2006)

10. Burchell, M.J., et al.: Cosmic Dust Collection in Aerogel. Annual Review of Earth and Planetary. Science 34, 385-418 (2006)

11. Carpenter, J.D., et al.: Dust Detection in the ISS environment using thin filmed microchannel plates. J. Geophys. Res., 110, E05013 (2005)

12. Carpenter, J.D. et al.: Nanometer hypervelocity dust impacts in low Earth orbit. J. Geophys. Res. 112, E08008 (2007) DOI 10.1029/2007JE002923

13. Cataldo, F.: Cyanopolyynes: carbon chains formation in a carbon arc mimicking the formation of carbon chains in the circumstellar medium. Int. J. Astrobiology 3, (03), 237-246 (2004)

14. Cernicharo, J., et al.: Infrared space observatory's discovery of $\mathrm{C}_{4} \mathrm{H}_{2}, \mathrm{C}_{6} \mathrm{H}_{2}$, and benzene in CRL 618 . Astrophys J. 546, (2), L123-L126 (2001)

15. Charnley, S.B., Markwick, A.J.: Complex bifurcations in interstellar chemistry? Astron. Astrophys. 399, 583-587 (2003)

16. Cherchneff, I., et al.: Polycyclic aromatic hydrocarbon formation in carbon-rich stellar envelopes. Astrophys. J. Part 1. 401, (1), 269-287 (1992)

17. Colangeli, L., della Corte, V., Esposito, F., Mazzotta Epifani, E., Palomba, E., Lopez-Moreno, J.J., Rodriguez, J., Morales, R., Lopez-Jimenez, A., Herranz, M., Moreno, F., Palumbo, P., Rotundi, A., 
Cosi, M. and The International Giada Consortium: "The GIADA experiment for the Rosetta mission" in The New Rosetta Targets. Observations, simulations and instrument performances. Edited by Luigi Colangeli, Elena Mazzotta Epifani, and Pasquale Palumbo. Astrophys. Space Sci. Libr. 311, 271-280 (2004)

18. Colangeli, L., Lopez Moreno, J.J., Palumbo, P., Rodriguez, J., Bussoletti, E., Della Corte, V., Esposito, F., Herranz, M., Jeronimo, J.M., Lopez-Jimenez, A., Mazzotta Epifani, E., Morales, R., Palomba, E., Rotundi, A., Vergara, S., the International GIADA Team: GIADA: The Grain Impact Analyser and Dust Accumulator for the Rosetta space mission. Adv. Space Res. 39, 446-450 (2007a)

19. Colangeli, L., Lopez-Moreno, J.J., Palumbo, P., Rodriguez, J., Cosi, M., Della Corte, V., Esposito, F., Fulle, M., Herranz, M., Jeronimo, J.M., Lopez-Jimenez, A., Epifani, E.M., Morales, R., Moreno, F., Palomba, E., Rotundi, A.: The Grain Impact Analyser and Dust Accumulator (GIADA) Experiment for the Rosetta Mission Design, Performances and First Results. Space Sci. Rev. 128, 803-821 (2007b)

20. Davies, J.K., Green, S.F., Stewart, B.C., Meadows, A.J., Aumann, H.H.: The IRAS fast-moving object search. Nature 309, 315-319 (1984)

21. Ehrenfreund P. and Fraser H., Ice chemistry in space, Solid state astrochemistry. Proceedings of the NATO Advanced Study Institute on Solid State Astrochemistry, Erice, Sicily, Italy, NATO Science Series II: Mathematics, Physics and Chemistry, vol. 120, pp. 317-356. Dordrecht: Kluwer Academic Publishers (2003) ISBN 1-4020-1558-5, 2003

22. Esposito, F., Colangeli, L., della Corte, V., Palumbo, P.: Physical aspect of an "impact sensor" for the detection of cometary dust momentum onboard the "Rosetta" space mission. Adv. Space Res. 29, 1159-1163 (2002)

23. FoingEhrenfreund, B.H.P.: Detection of two interstellar absorption bands coincident with spectral features of $\mathrm{C}_{60}$. Nature. 369, 296-298 (1994)

24. Foing, B.H., Ehrenfreund, P.: New evidences for interstellar C60. Astron. Astrophys. 317, L59-L62 (1997)

25. Frenklach, M., Feigelson, E.D.: Formation of polycyclic aromatic hydrocarbons in circumstellar envelopes. Astrophys. J. Part 1. 341, 372-384 (1989)

26. Gardner, D.J., et al.: Hole growth characterisation for hypervelocity impacts in thin targets. Int. J. Impact Eng. 19, (7), 589-602 (1997)

27. Graham, G.A., et al.: Observations on hypervelocity impact damage sustained by multi-layered insulation foils exposed in low earth orbit and simulated in the laboratory. Int J Impact Eng 29, 307316 (2003)

28. Green, S.F., McDonnell, J.A.M., McBride, N., Colwell, M.T.S.H., Tuzzolino, A.J., Economou, T.E., Tsou, P., Clark, B.C., Brownlee, D.E.: Comet 81P/Wild 2 dust mass distribution. J. Geophys. Res. 109, E12504 (2004)

29. Grün, E. et al.: Orbits of interplanetary dust particles inside 1 AU as observed by HELIOS, Properties and interactions of interplanetary dust; Proceedings of the Eighty-fifth Colloquium, Marseille, France, July 9-12, 1984 (A86-42326 20-90). Dordrecht, D. Reidel Publishing Co., 105-111 (1985)

30. Grün, E., et al.: The Galileo dust detector. Space Sci. Rev. 60, 317-340 (1992a)

31. Grün, E., et al.: The Ulysses dust experiment. Space Sci. Rev. 92, 411-423 (1992b)

32. Grün, E., Baguhl, M., Svedhem, H., Zook, H.A.: In situ measurements of cosmic dust. In: Grün E. (ed) Interplanetary Dust, pp. 295-346. Springer, London and Berlin (2001a)

33. Grün, E., Kempf, S., Krüger, H., Landgraf, M., Srama, R.: Dust Astronomy: A new approach to study the interstellar dust. In: Barbara Warmbein (ed), Proc. Of the Meteoroids 2001 Conf. 6-10, Aug 2001, Kiruna, Sweden, ESA SP-495, ESA Publications Division, Noordwijk, pp. 651-662. ISBN 92-9092805-0, (2001b)

34. Guillois, O., et al.: Diamond infrared emission bands in circumstellar media. Astrophys J. 521, (2), L133-L36 (1999)

35. Hörz, F., et al.: Impact features on Stardust and Comet Wild 2 dust. Science 314, 1716-1719 (2006)

36. Humayun, M., Cassen, P.: Processes determining the volatile abundances of the meteorites and terrestrial planets. In: Canup R.M., Righter K., et al. (eds) Origin of the earth and moon, pp 3-23. University of Arizona Press, Tucson (2000)

37. Jones, S.M., Flynn, G.J., Frank, D., Westphal, A.J.: Non-silicate aerogels as a next generation hypervelocity particle capture material. Lunar and Planetary Sci. XXXIX, 1445 (2008)

38. Kearsley, A.T. et al.: Sampling The Orbital Debris Population Using A Foil Residue Collector In A Standardised Container For Experiments (SCE). In: Danesy, D. (ed) Proceedings of the 4th European Conference on Space Debris, ESA Special Publication 587, pp 215-220. The European Space Agency, Darmstadt, Germany (2005a)

39. Kearsley, A.T., et al.: MULPEX: a compact multi-layered polymer foil collector for micrometeoroids and orbital debris. Adv. Space Res. 35, 1270-1281 (2005b) 
40. Kearsley, A.T., et al.: Analytical scanning and transmission electron microscopy of laboratory impacts on Stardust aluminum foils: Interpreting impact crater morphology and the composition of impact residues. Meteorit. Planet. Sci. 42.2, 191-210 (2007)

41. Kearsley A. T. et al.: Dust from comet Wild 2: interpreting particle size, shape, structure and composition from impact features on the Stardust aluminum foils. Meteorit. Planet. Sci., (in press) (2008)

42. Kempf, S., Srama, R., Altobelli, N., Auer, S., Tschernjawski, V., Bradley, J., Burton, M.E., Helfert, S., Johnson, T.V., Krüger, H., Moragas-Klostermeyer, G., Grün, E.: Cassini between Earth and asteroid belt: first in-situ charge measurements of interplanetary grains. Icarus 171, (2), 317-335 (2004)

43. Kissel, J., Krueger, F.: The organic component in dust from comet Halley as measured by the PUMA mass spectrometer on board VEGA 1. Nature 326, 755-760 (1987)

44. Kissel, J., et al.: The cometary and interstellar dust analyzer at Comet 81P/Wild 2. Science 304, (5678), 1774-1776 (2004)

45. Kroto, H.W., et al.: C(60): Buckminsterfullerene. Nature 318, 162-163 (1985)

46. Kuan, Y.J., et al.: A search for interstellar pyrimidine. Mon. Not. R. Astron. Soc. 345, (10), 650-656 (2003)

47. Leitner, J., Stephan, T., Kearsley, A.T., Hörz, F., Flynn, G.J., Sandford, S.A.: TOF-SIMS analysis of crater residues from Wild 2 cometary particles on Stardust aluminum foil. Lunar Planet. Sci. XXXVIII, 1591 (2007)

48. Mann, I., Murad, E., Czechowski, A.: Nanoparticles in the inner solar system. Planet. Space Sci. 55, $1000-1009$ (2007)

49. McDonnell, J.A.M., et al.: Physical properties of cometary dust. Comets in the post-Halley era, vol 2 , pp. 1043-1073. Kluwer, Dordrecht (1991)

50. McDonnell J.A.M. et al.: Near Earth environment. In: E. Grün (ed) Interplanetary Dust, pp 163-261. Springer, London and Berlin (2001)

51. McKeegan, K.D., et al.: Isotopic compositions of cometary matter returned by Stardust. Science 314, 1724-1728 (2006)

52. Messenger, S, Walker, R.M.: Evidence for molecular cloud material in meteorites and interplanetary dust, astrophysical implications of the laboratory study of presolar materials. St. Louis, MO, October 1996. Bernatowicz TJ., Zinner E. (eds). American Institute of Physics, Woodbury, N.Y.. c1997., 545 (1997)

53. Mumma, M.J.: Organic Volatiles in Comets:Their Relation to Interstellar Ices and Solar Nebula Material, From Stardust to Planetesimals. Symposium held as part of the 108th Annual meeting of the ASP held at Santa Clara, California 24-26 June 1996. ASP Conference Series, vol. 122. 1997, 369, 1997

54. Nuth, J.A.: Meteoritic evidence that graphite is rare in the interstellar medium. Nature 318, 166-168 (1985)

55. de Pater, I., Lissauer, J.J.: Planetary Sciences, p. 544. Cambridge University Press, Cambridge UK (2001) ISBN 0521482194

56. Rost D., Stephan, T., Vicenzi E.P., Bullock E.S., MacPherson G.J., Westphal A.J., Snead C.J., Flynn G.J., Sandford S.A., Zolensky M.E.: TOF-SIMS analysis of cometary matter in Stardust aerogel tracks. Lunar Planet. Sci. XXXVIII, 2346 (2007)

57. Sephton, M.A., Botta, O.: Recognizing life in the solar system: guidance from meteoritic organic matter. Int. J. Astrobiol. 4, (3), 269-276 (2005)

58. Simpson, J.A., Tuzzolino, A.J.: Polarized polymer films as electronic pulse detectors of cosmic dust particles. Nucl. Inst. Meths. Sect. A. 236, 187-202 (1985)

59. Simpson, J.A., Tuzzolino, A.J.: II. Instruments for measurement of particle trajectory, velocity and mass. Nucl. Inst. And Meths. Sect. A. 279, 611-624 (1989)

60. Srama, R., et al.: The Cassini cosmic dust analyser. Space Sci. Rev. 114, 465-518 (2004)

61. Srama, R. et al., Performance of an Advanced Dust Telescope, Proceedings of the 4th European Conference on Space Debris (ESA SP-587). 18-20 April 2005, ESA/ESOC, Darmstadt, Germany. Editor: D. Danesy., p.171, (2005a)

62. Srama, R., et al.: Development of an advanced dust telescope. Earth Moon Planets 95, (1-4), 211-220 (2005b)

63. Srama, R., Kempf, S., Moragas-Klostermeyer, G., Landgraf, M., Helfert, S., Sternovsky, Z., Rachev, M., Grün, E.: Laboratory tests of the large area mass analyzer, Proc. of "Dust in Planetary Systems", Kauai, Hawaii, USA, ESA SP-643, 209-213 (2007)

64. Stadermann, F.J. et al.: Stardust in STARDUST - the C, N, and O isotopic compositions of Wild 2 cometary matter in Al foil impacts. Meteorit. Planet. Sci. (in press) (2008)

65. Stephan, T., et al.: TOF-SIMS analysis of Allende projectiles shot into silica aerogel. Meteorit. Planet. Sci. 41, 211-216 (2006a)

66. Stephan, T., et al.: TOF-SIMS analysis of aerogel picokeystones - an analogue to Stardust's interstellar dust collection. Lunar Planet. Sci. 37, 1448 (2006b) 
67. Sternovsky, Z., et al.: The Large Area Mass Analyser (LAMA) for in-situ Chemical Analysis of Interstellar Dust Particles, Proc. of "Dust in Planetary Systems", Kauai, Hawaii, USA, ESA SP-643, 205-209 Jan. 2007

68. Tsou, P., et al.: Wild 2 and interstellar sample collection and Earth return. J. Geophys. Res. 118, 8113 (2003)

69. Tuzzolino, A.J.: Applications of PVDF dust sensor systems in space, Adv. Space Res. 17, 123-132 (1996)

70. Tuzzolino, A.J., et al.: Dust Flux Monitor Instrument for the Stardust mission to comet Wild 2, J. Geophysical Res. 108, (E10), 8115 (2003)

71. Westphal, A.J., et al.: Aerogel keystones: Extraction of complete hypervelocity impact events from aerogel collectors. Meteorit. Planet. Sci. 39, 1375-1386 (2004)

72. Zolensky, M.E., et al.: Mineralogy and petrology of Comet 81P/Wild 2 nucleus samples. Science 314, (5806), 1735-1739 (2006) 